\title{
Perception of sentences in noise using different number of channels in simulated cochlear implant listeners
}

\begin{abstract}
Introduction: Previous literature reported increase in speech perception with increase in number of electrodes among individuals with cochlear implant.

Objective: The objective of the study is to focus on the perception of sentences using different number of channels and signal to noise ratios (SNRs) in simulated cochlear implant listeners.

Methods: The study comprised of thirty adults with normal hearing. Angelsim program was used to frame 4 sentence list which comprised of 40 sentences at +5.0 and $-5 \mathrm{~dB}$ SNR across different channels ( 4,8 and 32 channel). The recorded stimuli was presented in free field condition in which the situation varied from more most adverse situation (4 channels, $-5 \mathrm{dBSNR}$ ) to least adverse listening situation (32 channels, $+5 \mathrm{dBSNR}$ ) through a computer and a CD player which was routed through speakers placed at 00 azimuth.
\end{abstract}

Results: Statistical analysis was carried out in which repeated measures of ANOVA revealed that there is considerable main effect across various SNRs at 4, 8 and 32 channel. The Bonferroni multiple pairwise comparisons also showed that there is significant difference in the nature of perceiving sentences with different channels $(4,8,32$ channels $)$ at $+5 \mathrm{~dB}$, $0 \mathrm{~dB}$ and $-5 \mathrm{~dB}$ SNR.

Conclusion: The finding of the current investigation enlightens the importance of greater number of channels and higher signal to noise ratio for enhanced perception of sentence in the presence of noise in simulated condition.
Volume 8 Issue 4 - 2017

\author{
Himanshu Kumar Sanju,' Manisha \\ Choudhury, ${ }^{2}$ Prawin Kumar $^{3}$ \\ 'Department of Audiology and Speech Language Pathology \\ Amity Medical School Amity University, India \\ ${ }^{2}$ Speech Language Pathologist Department of Audiology and \\ Speech Language Pathology Amity Medical School Amity \\ University, India \\ ${ }^{3}$ Reader in Audiology All India Institute of Speech and Hearing, \\ India
}

Correspondence: Himanshu Kumar Sanju Assistant Professor Department of Audiology and Speech Language Pathology Amity University Haryana, India, Tel +9l-7406093279,

Email himanshusanjul I2@gmail.com

Received: June 07, 2017| Published: September 07, 2017

\section{Introduction}

Previous literature in cochlear implant has revealed that ability to recognize speech increases with the corresponding increase number of channels. ${ }^{1-3}$ Various studies have also reported that speech perception gets improved with increase in number of channels in quite listening condition to the extent 4 to 7 channels. ${ }^{4,5}$ Fishman et al., ${ }^{6}$ investigated perception of speech in subjects with the help of Nucleus-22 SPEAK speech processing strategy with increase in number of electrodes. Result showed deprived performance in all the subjects using single electrode processor whereas the average performance improved over all the test materials as the number of electrode increased from 1 to 4. They also observed that on an average performance there was no difference found on any test in 7-, 10-, and 20-electrode conditions. Similarly, Friesen et al., ${ }^{7}$ in 2001 measured speech recognition using consonant, vowel, word, and sentence in 24 listeners. Among those 24 listeners, 5 normal hearing, 10 subjects with Nucleus 22 and 9 with Advanced Bionics Clarion. In their study, speech perceptions were measured with increase in number of electrodes and signal to noise ratio. They reported that as the electrode number added up to 7 or 8 , the performance increased. They also found that for all noise levels, with SPEAK speech processor, vowel and consonant recognition did not enhance after seven electrodes. Whereas the performance continued to improve till 20 channels for normal hearing listeners. In Nucleus 22 cochlear implant listeners, with the increase in electrode numbers from 7 to 10 , the recognition of speech was found to be significantly improved even over speech materials with greater difficulty which comprises of words and sentences. Verschuur ${ }^{8}$ in 2009 also had conducted similar kind of research in which he observed that consonants perception had improved with increase in number of channels in users with Nucleus 24. In a similar line, Friesen et al., ${ }^{3}$ in 2009 investigated test on speech recognition with CVC stimuli at various spectral channels such as $2,4,8,12$, and 16 on 10 subjects with normal hearing. Results revealed that as the number of spectral channels increased the perception of CVC was enhanced. Perreau et al., ${ }^{9}$ in 2010 measured speech recognition performance in spatially separated noise with different number of channels. They observed that the performance of the individuals was affected when the number of electrodes was reduced. Zeitler et al., ${ }^{10}$ in 2009 through his study studied speech perception outcomes by decreasing quantity of functional electrodes post implantation. The finding of the study revealed that even after deactivation of electrodes didn't have any straight effect on speech perception, but loss of 5 or more electrodes indicated imminent failure of the device. From the above stated literature it can be inferred that many of these studies were conducted in quiet and feasible hearing conditions, whereas in our day to day life situations we cannot avoid the presence of background noise. So, there is an importance to take up a study that could reveal the effect of various SNRs in recognition of sentence at various numbers of channels present in a cochlear implant in different simulated environments. The aim of the current investigation is to explore the effect of various SNRs on perception of sentences at various numbers of channels.

\section{Methods}

\section{Participants}

The study included 30 individuals ranging from age 18 to 25 years (mean age 22.52years) with normal hearing. Individuals included in the study were made sure to have hearing sensitivity within normal 
limits as indicated by thresholds on pure tones of $\leq 15 \mathrm{dBHL}$ at 250 $\mathrm{Hz}$ to $8000 \mathrm{~Hz}$ for air conduction. Further, the subjects were ensured for normal middle ear function using middle ear analyzer. To ensure normal outer hair cells functioning, TEOAE (transient oto-acoustic emission) was performed on all subjects. Subjects with outer hair cells dysfunctioning were excluded from the study. Auditory brainstem response for site of lesion was also done on all subjects to rule out retrocochlear pathology. Those subjects who were suspected to have otological, neurological and/or neuromuscular issues were removed from the participants list of the study.

\section{Testing environment}

Sound treated room was used to carry out all the behavioral tests where ANSI S3.1 (1999) prescribed levels of noise were ensured. Well illuminated and air conditioned room were used to carry out tests for ensuring the comfort of the participants of the study.

\section{Instrumentation}

Calibrated audiometer (Piano Inventis) with two channels was used for evaluating pure tone threshold and perception of sentence at different number of electrodes and various SNRs. Calibrated GSITympstar middle ear analyzer was used for carrying out tympanometry and reflexometry. TEOAE was done with ILO (Version 6). Auditory brainstem response for site of lesion was carried out using Intelligent Hearing System (Smart EP).

\section{Procedure}

Pure tone thresholds across frequencies from 250 to $8000 \mathrm{~Hz}$ for air conduction and from 250 to $4000 \mathrm{~Hz}$ for bone conduction were acquired using modified Hughson and Westlake method given by Carhart \& Jerger. ${ }^{11}$ Immittance meter (GSI-Tympstar) was used to check middle ear function. Using this instrument, tympanogram at $226 \mathrm{~Hz}$ probe frequency and acoustic reflexes from $500 \mathrm{~Hz}$ to 4000 $\mathrm{Hz}$ can be obtained. Presence of TEOAEs revealed normal outer hair cells functioning in all participants. Normal measures for auditory brainstem response for site of lesion revealed no indication of retrocochlear pathology for all selected participants. For perception of sentence test, Angelsim program was used to process 4 sentence list comprising of 40 sentences over different channels (4,8 and 32 channel) at $+5,0$ and $-5 \mathrm{dBSNR}$. Each sentence list was consisting of 10 sentences. Clear instructions were given to the subjects to write down the sentences and they were motivated to predict the sentences. Feedback and repetition of sentences were not allowed. Subjects were made sure to sit comfortably and avoid excessive movements of head. Adequate lightening and suitable temperature in the room was maintained for comfort of the participants. Testing procedure followed a sequence of testing from most adverse situation (4 channels, -5dBSNR) to least adverse listening situation (32 channels, $+5 \mathrm{dBSNR}$ ). After completing every channel condition rest period was provided to the participants. The recorded sentences were presented in free field condition through a computer with $C D$ player routed through an audiometer through speakers placed at 00 azimuth in a well sound treated room. At the testing procedure carried out in a sound treated room the subject was made sure to be seated comfortably at a distance of 1 meter from the loudspeaker. The stimuli were presented at 3 various SNR's $(-5 \mathrm{~dB}, 0 \mathrm{~dB} \&+5 \mathrm{~dB})$ by altering the level of speech noise (generated by PIANO Inventis audiometer), placing the level of signal at $40 \mathrm{dBSPL}$ in various simulated channels i.e. 4,8 and 32 channels. The participants were instructed to write down the sentences presented to the subjects through free field condition. Raw scores were calculated for sentences.

\section{Results}

To examine the collected data from the participants, bonferroni multiple pairwise, repeated measure ANOVA and descriptive statistics comparisons were done with the help of SPSS 17. To find mean and standard deviation of score at different number of channels with at various SNRs descriptive statistics was done by the investigators. Repeated measure ANOVA was carried out to investigate any significant main effect across various SNRs i.e. 4, 8 and 32 channels. Bonferroni multiple pairwise comparisons was carried out to check any considerable differences between all the possible combinations (4 channel, 8 channel, and 32 channel) at $-5 \mathrm{~dB}$ SNR, $0 \mathrm{~dB}$ SNR and $+5 \mathrm{~dB}$ SNR.

Descriptive statistics revealed that mean score improved (better) with increment in number of channels for sentences (Table 1). In similar line descriptive statistics also showed that mean score improved (better) with increase in SNR i.e. $-5,0$ to $+5 \mathrm{~dB}$ for sentences (Table 2 ). The repeated measure ANOVA presented considerable main effect across various SNRs at 4 channel $F(2,87)=230.97 ; p<0.05$; Partial eta squared $=0.842 ; 8$ channel $F(2,87)=370.25$; $<<0.05$; Partial eta squared $=0.895$; and at 32 channel $F(2,87)=204.73 ; \mathrm{p}<0.05$; Partial eta squared $=0.825$. Additional, Bonferroni multiple pairwise comparisons shows significant differences between all the possible combinations (4 channel, 8 channel, and 32 channel) at $+5 \mathrm{~dB}$ SNR, $0 \mathrm{~dB}$ SNR and $-5 \mathrm{~dB}$ SNR (Table 2). Error bar graph of mean score for sentences with 4,8 and 32 channels at $+5 \mathrm{~dB}, 0 \mathrm{~dB}$ and $-5 \mathrm{~dB}$ SNR is shown in Figure 1.

Table I Mean and standard deviation of only correct raw score (sentences) with various numbers of channels at different SNRs

\begin{tabular}{|c|c|c|c|c|c|c|}
\hline & $\begin{array}{l}-5 \mathrm{~dB} \\
\text { SNR }\end{array}$ & & $\begin{array}{l}\text { OdB } \\
\text { SNR }\end{array}$ & & $\begin{array}{l}+5 \mathrm{~dB} \\
\text { SNR }\end{array}$ & \\
\hline & Mean & SD & Mean & SD & Mean & SD \\
\hline 4 Channels & 0.5 & 0.77 & 0.96 & 1.09 & 1.96 & 1.86 \\
\hline 8 Channels & 4.36 & 1.88 & 5.43 & 1.35 & 6.66 & 1.42 \\
\hline 32 Channels & 8.43 & 1.4 & 8.96 & 0.92 & 9.3 & 0.74 \\
\hline
\end{tabular}

Table 2 Bonferroni multiple pairwise comparisons at every possible combination for sentence ( 4 channel, 8 channel, and 32 channel) at $+5 \mathrm{~dB}$ SNR, 0 dB SNR and $-5 \mathrm{~dB}$ SNR, $(* * \mathrm{p}<0.00 \mathrm{I})$

\begin{tabular}{llll}
\hline SNR & Channels & $\mathbf{8}$ Channels & 32 Channels \\
\hline & & $\begin{array}{l}\text { (Mean } \\
\text { Difference) }\end{array}$ & $\begin{array}{l}\text { (Mean } \\
\text { Difference) }\end{array}$ \\
\hline$+5 \mathrm{dBSNR}$ & 4 Channels & $-4.70^{* *}$ & $-7.33^{* *}$ \\
& 8 Channels & & $-2.63^{* *}$ \\
0 dBSNR & 4 Channels & $-4.46^{* *}$ & $-8.00^{* *}$ \\
& 8 Channels & & $-3.53^{* *}$ \\
$-5 \mathrm{dBSNR}$ & 4 Channels & $-3.86^{* *}$ & $-7.93^{* *}$ \\
& 8 Channels & & $-4.06^{* *}$ \\
\hline
\end{tabular}

\section{Discussion}

The current study discovered the effect of various numbers of channels on perception of sentence at different signal-to-noise ratios (SNRs). The outcome revealed significant betterment in recognition of sentence when the number of channels was increased. Present study also revealed considerable enhancement in perception of sentence when the SNRs was increased at different number of channels. Through current study, researchers are also able to quantify the damaging effect on understanding of sentences with proceeding decrement in SNR at various channels. The outcome of the current investigation is in a similar line with preceding literature. ${ }^{1,3,7,9,10,12}$ However, there are few studies contradict the findings of the present study. ${ }^{6,8}$ As per the present study results which indicate that sentence perception increases with 
increase in number of channels ( 4 channels $<8$ channels $<32$ channels) and of favorable SNR $(-5 \mathrm{dBSNR}<0 \mathrm{dBSNR}<+5 \mathrm{dBSNR})$. However, to achieve atleast more than $50 \%$ performance minimum 8 channels are required regardless of adversarial listening condition (-5dBSNR). Perhaps information content is spectrally sparse at lesser number of channels and hence performance is poorer which is observed to detoriate in adverse listening conditions (-5dBSNR). The results of current study also disclosed damaging effect of noise exists even at (most favorable) 32 channels condition. Similarly, these results measure the effect of number of spectral channels on perception of speech in noise and most of the cochlear implant users found difficulty in utilizing the speech information that are supplied by the number of electrode present in the implant. Friesen et al., ${ }^{7}$ assessed recognition of speech in both noise (at $+15,+10,+5,0 \mathrm{~dB}$ ) as well as quiet situations at different number of electrode. The performance was improved when the number of electrodes was increased up to seven or eight. When difficult speech materials consisting words and sentences were used, the recognition of speech score revealed marginal improvement in Nucleus 22 users with 7 to 10 electrodes. Liu et al.13 in 2004 assessed Mandarian tone perception by altering the number of programmed electrode in 24 children using Nucleus cochlear implant. Result indicates little but significant reduction in Mandarin tone perception was found as the numbers of active electrodes were decreased in subjects using CI 24 cochlear implants. Perreau et al., ${ }^{9}$ in 2010 also studied the consequence of decreasing the electrodes number in subjects using cochlear implant bilaterally. The finding of the study showed that a minimum of 3-4 electrodes can be sufficient to get maximum results on speech-in-noise tests.

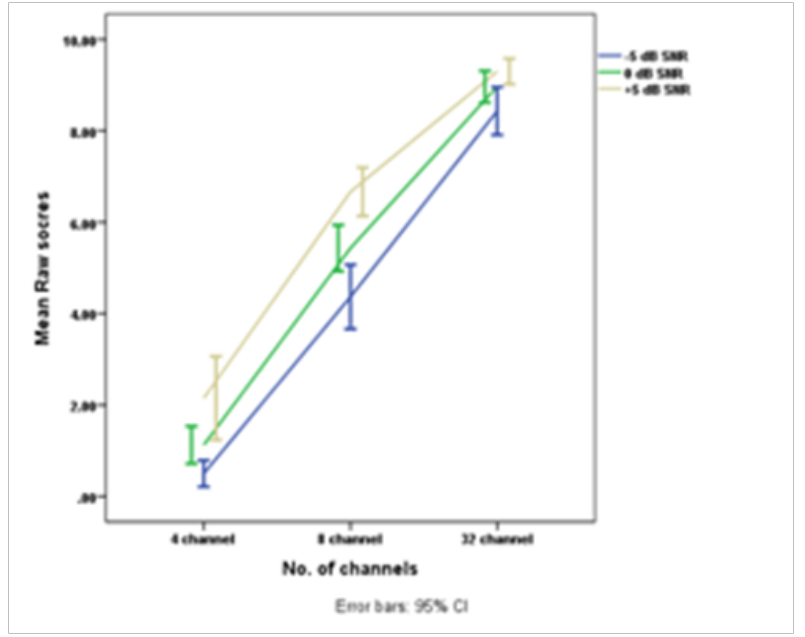

Figure I Error bar graph of mean score for sentences with 4, 8 and 32 channels at $+5 \mathrm{~dB}, 0 \mathrm{~dB}$ and $-5 \mathrm{~dB}$ SNR.

However, other participants exhibited a gradual decrease in performance on speech-in-noise tests when the number of electrodes reduced. Zeitler et al. ${ }^{10}$ in 2009 examined speech recognition ability as related to a reduction in the number of functional electrodes postimplantation. The result also revealed that there was no direct effect on the speech perception performance, the loss of 5 or more electrodes showed impending failure of device. The finding of the present study is in contrary with the study carried out by Fishman et al., ${ }^{6}$ revealed that there is no difference found in an average performance among any test in the seven, ten and twenty electrode situations. Their finding also reported no difference between sentence and consonant test performance with 4 and 20 electrode processor. Similarly, Verschuur ${ }^{8}$ in 2009 also reported that great changes in number of electrodes in the Advanced Combination Encoder (ACE) signal processing strategy lead to no significant changes in performance. In contrary, Kumar et al., ${ }^{2}$ showed the importance of more number of channels and higher signal to noise ratio in word perception which is in similar line with the findings of present study. However, the current study was carried out on simulated cochlear implantees, professional (audiologist) or clinical audiologist requests to be aware before administer current finding on subjects with cochlear implant.

\section{Conclusion}

The results of the current study enlightens the importance of more number of channels and favorable SNR levels for better perception of sentence in presence of noise in simulated cochlear implant condition. The finding of present study also showed that to achieve atleast more than $50 \%$ performance, minimum 8 channels are mandatory regardless of adversarial listening conditions.

\section{Acknowledgments}

None.

\section{Conflicts of interest}

Author declares there are no conflicts of interest.

\section{Funding}

None.

\section{References}

1. Eddington DK, Rabinowitz WR, Tierney J, et al. Speech Processors for Auditory Prostheses. 8th Quarterly Progress Report, USA. 1997.

2. Kumar P, Sanju HK, Kumar S, et al. Word perception in noise at different channels in simulated cochlear implant listeners. World $J$ Otorhinolaryngol. 2016;6(2):45-49.

3. Friesen LM, Tremblay KL, Rohila N, et al. Evoked cortical activity and speech recognition as a function of the number of simulated cochlear implant channels. Clin Neurophysiol. 2009;120(4):776-782.

4. Dorman MF, Loizou PC, Rainey D. Simulating the effect of cochlearimplant electrode insertion depth on speech understanding. J Acoust Soc Am. 1997;102(5 Pt 1):2993-2996.

5. Fu QJ, Shannon RV, Wang X. Effects of noise and spectral resolution on vowel and consonant recognition: acoustic and electric hearing. $J$ Acoust Soc Am. 1998;104(6):3586-3596.

6. Fishman KE, Shannon RV, Slattery WH. Speech recognition as a function of the number of electrodes used in the SPEAK cochlear implant speech processor. J Speech Lang Hear Res. 1997;40(5):1201-1215.

7. Friesen LM, Shannon RV, Baskent D, et al. Speech recognition in noise as a function of the number of spectral channels: comparison of acoustic hearing and cochlear implants. J Acoust Soc Am . 2001;110(2):1150-1163.

8. Verschuur C. Modeling the effect of channel number and interaction on consonant recognition in a cochlear implant peak-picking strategy. $J$ Acoust Soc Am. 2009;125(3):1723-1736.

9. Perreau A, Tyler RS, Witt SA. The effect of reducing the number of electrodes on spatial hearing tasks for bilateral cochlear implant recipients. J Am Acad Audiol. 2010;21(2):110-120.

10. Zeitler DM, Lalwani AK, Roland JT, et al. The effects of cochlear implant electrode deactivation on speech perception and in predicting device failure. Otol Neurotol. 2009;30(1):7-13.

11. Carhart R, Jerger J. Preferred method for clinical determination of pure-tone thresholds. Journal of Speech and Hearing Disorders. 1959;24(4):330-335. 
12. Lawson DT, Wilson BS, Zerbi M. Speech processors for auditory prostheses. Third Quarterly Progress Report. Neuroscience Program Office Research Triangle Institute Research Triangle Park, NC, USA. 1993.
13. Liu TC, Chen- HP, Lin HC. Effects of limiting the number of active electrodes on Mandarin tone perception in young children using cochlear implants. Acta Otolaryngol. 2004;124(10):1149-1154. 